\title{
The correlation between pancreatic dysfunction markers and selected indices of periodontitis
}

\author{
Andrzej Miskiewicz, ${ }^{1, A-F}$, Grzegorz Szparecki' ${ }^{2, B-D}$, Marek Durlik ${ }^{3, A, B, D, E}$, \\ Grażyna Rydzewska ${ }^{4, A, B, D, E}$, Ireneusz Ziobrowski ${ }^{3, A, B, E}$, Renata Górska ${ }^{1, A-F}$ \\ ${ }^{1}$ Department of Periodontology and Oral Diseases, Medical University of Warsaw, Poland \\ 2 Department of Pathology, Medical University of Warsaw, Poland \\ ${ }^{3}$ Department of Gastroenterological Surgery and Transplantation, Central Clinical Hospital of the Ministry of Internal Affairs, Warszawa, Poland \\ ${ }^{4}$ Department of Internal Medicine and Gastroenterology with Inflammatory Bowel Diseases Unit, Central Clinical Hospital of the Ministry of Internal Affairs, Warszawa, Poland \\ A - research concept and design; B - collection and/or assembly of data; $\mathrm{C}$ - data analysis and interpretation; \\ $D$ - writing the article; $E$ - critical revision of the article; $F$ - final approval of the article
}

\section{Address for correspondence}

Andrzej Miskiewicz

E-mail: andrzej.miskiewicz@wum.edu.pl

Funding sources

The research was funded by the authors' institution, the Medical University of Warsaw, Poland (grant No. 1S14/NM1/13).

Conflict of interest None declared

Received on March 31, 2016

Reviewed on July 1,2016

Accepted on September 1, 2016

\begin{abstract}
Background. Periodontitis is a major risk factor of systemic diseases with inflammatory etiology. Numerous studies have established the connection between periodontal condition and diabetes, but there are no reports in the literature on the relationship between periodontitis and other pancreas conditions. The activity of pancreatic enzymes is an important parameter of pancreatic damage, and is also a diagnostic marker of acute and chronic pancreatitis, and the bleeding index determines the periodontitis activity. To date, the periodontal status in both of the above-mentioned conditions has not been examined.
\end{abstract}

Objectives. The objective of the study was to provide a clinical evaluation of oral cavity hygiene and the condition of periodontal tissues in patients diagnosed with pancreatic adenocarcinoma or chronic pancreatitis and in the control group, and to assess the correlation between the activity of periodontitis and the concentration of glycated hemoglobin $\mathrm{HbA}_{\mathrm{k}}$ lipase activity and pancreatic amylase in serum from the examined groups.

Material and methods. The serum activity levels of amylase, lipase and $\mathrm{HbA}_{\mathrm{I}}$ concentration were correlated with periodontitis activity markers in patients diagnosed with chronic pancreatitis $(n=41)$, pancreatic cancer $(n=29)$ and in the control group $(n=50)$.

Results. In the group with chronic pancreatitis, we have found a positive association between the bleeding on probing (BOP) and the amylase activity $(r=0.64)$ as well as the lipase $(r=0.62 ; p<0.05)$. The patients diagnosed with pancreatic cancer evinced a higher value of the inflammatory response index, namely 1.51, than the other studied groups $(H=24.94 ; p=0.01)$. Patients diagnosed with chronic pancreatitis evinced the highest periodontitis activity indices: BOP: 62.53\% $(H=61.31 ; p=0.01)$ and probing depth (PD): $4.14 \pm 0.89 \mathrm{~mm}(H=22.43 ; p<0.0001)$

Conclusions. The study showed that periodontitis in patients with pancreatic cancer is independent of the state of oral hygiene. In the group of patients with chronic pancreatitis, the observed positive correlation between the bleeding index and the enzyme activity suggests interaction between the examined diseases.

Key words: chronic pancreatitis, periodontitis, pancreatic cancer

DOI

10.17219/acem/64937

Copyright

Copyright by Author(s)

This is an article distributed under the terms of the

Creative Commons Attribution Non-Commercial License

(http://creativecommons.org/licenses/by-nc-nd/4.0/) 


\section{Introduction}

Pancreatic adenocarcinoma is the $4^{\text {th }}$ and $6^{\text {th }}$ most common cause of death in the USA and Europe, respectively, among cancer-caused deaths. ${ }^{1}$ The 5 -year survival rate occurs in $6 \%$ of cases. $^{2}$ Therefore, when the final diagnosis is made, the neoplastic cells are no longer confined to the pancreas only, but are also present in other body organs, which in turn rules out the possibility of surgical intervention. The recent clinical reports state that the patient's survival rate in cases where surgery on pancreatic tumors is possible has not increased. ${ }^{3}$ However, if performed in the early stages, on less than $2 \mathrm{~cm}$ primary tumor size, the surgery may significantly reduce the death risk ratio $(\mathrm{HR}=1.41)$. A global effort is, therefore, currently being made in order to elaborate pancreatic cancer markers to help detect pancreatic cancer cases at a much earlier stage. On the other hand, exacerbation of chronic pancreatitis as well as first episodes of acute pancreatitis are among the most common reasons for gastrointestinal hospitalization emergencies. ${ }^{4}$ Additionally, chronic pancreatitis severely deteriorates the quality of the patient's life causing constant and recurring pain, unexpected exacerbations, and eventually leads to the patient's exocrine dysfunction. One of the most common problems faced by the currently conducted medical research is the neoplastic transformation occurring in the cases of ongoing chronic pancreatitis. Clinical signs of pancreatic deficiency, such as the afore-mentioned chronic abdominal pains, diabetes and malnutrition, are common for pancreatic cancer as well as for chronic pancreatitis. Moreover, both conditions modulate their course. Therefore, a case of an already diagnosed chronic pancreatitis does not rule out the possibility of an accompanying case of a developed neoplasm. According to earlier studies conducted and published by Zhang et al. and Wong et al., nucleic acid may be detected in the resting saliva and gingival crevicular fluid in patients suffering from pancreatic cancer and chronic pancreatitis. ${ }^{5,6}$

The most frequent marker determined in serum in the case of suspected pancreatitis is amylase. In the cases of acute pancreatitis or acute exacerbation of chronic pancreatitis, its activity increased above 1000 IU/L indicates, with a sensitivity of up to $95 \%$, exocrine pancreatic dysfunction (exocrine insufficiency). In addition, determination of serum lipase activity increases the sensitivity of detection of the disease. The level of $600 \mathrm{IU} / \mathrm{L}$ is adopted as the cut-off point indicating pancreas damage. Increased levels of the activity of these pancreatic enzymes also occur in the course of cancer of the salivary glands, stomach, intestines, and lungs. There are currently no studies reporting the correlation between the activity of these enzymes and periodontitis markers. Exocrine damage can mutually present with endocrine dysfunction, leading to impaired glucose tolerance and diabetes. One of the markers of glycemic control is glycated hemoglobin. So far, the involvement of these factors in patients with pancreatic cancer, developed in the course of chronic inflammation of this organ, has not been examined.

Periodontitis is caused by a wide spectrum of conditions affecting tissues surrounding a tooth. The clinical activity of the inflammatory periodontal process leads to alveolar bone osteolysis and connective tissue attachment loss. The course of the disease is modulated by pancreatic conditions, especially diabetes, which affects the immunological system of the host. ${ }^{7}$ Moreover, if left untreated, diabetes may modulate the course of inflammation in the periodontium, which is measured by Il-1 $\beta$ and $\beta$-glucuronidase concentration. ${ }^{8}$ Despite ample research carried out in this field, no clear correlation between periodontal activity markers and pancreatic dysfunction has been proven yet. The studies cited above made an attempt at assessing the occurrence of cytokines, enzymes and bacterial titres in patients with pancreatic conditions; however, no periodontitis activity parameters have been taken into consideration in those studies.

\section{Material and methods}

The study was conducted in the Central Clinical Hospital of the Ministry of Internal Affairs in Warszawa, Poland from April 2012 to August 2015.

In the study, we took into account 3 groups of individuals: patients diagnosed with pancreatic adenocarcinoma $(\mathrm{n}=29)$; chronic pancreatitis patients $(\mathrm{n}=41)$ and a control group $(n=50)$. The study groups were matched in line with the age of the participants, their gender and race, so that the data to be collected could be used to make a reliable comparison using relevant statistical tests.

The patients enrolled in the $1^{\text {st }}$ study group were patients of the Gastroenterological Surgery Ward diagnosed with pancreatic adenocarcinoma. The diagnosis was made initially by means of standard radiographic techniques and was subsequently confirmed following the surgery by means of a histopathological examination. The group consisted of 29 patients at the age of 41-81 years (median: 59.9 years). All admitted patients were referred to the ward upon an initial diagnosis of resectable pancreatic tumor, made based upon the criteria published by Wong et al. with the use of a computed tomography. ${ }^{9}$ The periodontal examination was performed 1 day before the planned surgery. Edentulous patients as well as those who had fewer than 20 teeth, used removable prosthetic devices, showed signs of fungal infections, or did not brush their teeth at least twice a day were all excluded from this group. Furthermore, patients with pancreatic tumor in stage III and IV were excluded - the criteria adapted were the involvement of the celiac axis or the superior mesenteric artery, portal vein or superior mesenteric vein. The history of antibiotics therapy in the past 3 months or chemotherapy/radiotherapy in the past 2 years were also criteria for exclusion. 
The $2^{\text {nd }}$ group consisted of 41 patients from the Gastroenterology Ward of the hospital mentioned above diagnosed with chronic pancreatitis, at the age of 20-87 years (median: 48.2 years). As for the newly diagnosed patients undergoing hospitalization in the ward for the first time, the diagnosis was confirmed in line with the TIGARO scale according to Etemad and Whitcomb. ${ }^{10}$ The exacerbations in the ongoing chronic pancreatitis patients were diagnosed using standard laboratory tests and radiographic techniques. The most common complaints among patients included in this group were newly occurring or exacerbated abdominal pains. From this group we excluded patients with alcohol abuse history as well as tobacco smoking patients. In addition, we used the same dental criteria for exclusion as in group 1.

The control group consisted of 50 patients of the Gastroenterology Ward, Department of Internal Medicine and Endocrinology Clinic. The age range was 29-69 years (median: 45.6 years). The probands selected to this group evinced no clinically relevant conditions regarding the digestive system organs. The inclusion to the group was made based on computed tomography and/or abdominal ultrasonography in order to exclude signs of pancreatic conditions including enlarged head of pancreas, and a dilated pancreatic duct and common bile duct. ${ }^{11}$ Moreover, to avoid bias in the study, we excluded patients with microlithiasis, the sphincter of Oddi dysfunction and cystic fibrosis. ${ }^{12}$ In this way we selected a group of probands among the hospitalized patients who were free of pancreatic and bile duct disorders. The periodontal examination was performed subsequently if the proband met the criteria in order to exclude the influence of the proposed treatment.

The study was carried out based on the consent issued by the Bioethical Committee of the Medical University of Warsaw on $25^{\text {th }}$ April, 2012. The study was conducted with the understanding and written consent of each enrolled patient. The patients included in the study agreed to participate in it by signing the informed consent form approved by the University Board of Ethics.

\section{Periodontal examination}

All included in the study probands underwent a dental and periodontal examination. It encompassed dental anamnesis concerning previous dental treatment, oral fungal infections, use of oral antibiotics, and everyday oral hygiene procedures. All the included patients brushed their teeth at least twice a day with toothpaste containing fluoride. Periodontal examination was performed by 1 dentist using a WHO 621 calibration dental probe. The measurements were performed in 6 diagnostic locations around every present tooth. According to the American Academy of Periodontology (AAP) classification of periodontal disease, we measured the following periodontal parameters:

- the plaque index (PI), measured on all surfaces of each tooth, according to a simplified scale by O'Leary: the proportion of surfaces covered with dental plaque to all examined surfaces [\%];

- the bleeding on probing index (BOP), measured as the percentage of bleeding sites around of each toot [\%], according to Ainamo's scale;

- the probing depth (PD), the distance measured between the marginal border of the gingiva and the periodontal pocket bottom (a WHO 621 calibration dental probe);

- the connective tissue attachment loss (CAL) level, measured by the distance between the cementoenamel junction and the periodontal pocket bottom. ${ }^{13}$

All the gathered data was included in the documentation and analyzed according to the AAP diagnosis criterion guidelines.

\section{Statistical analysis}

The numerical variables were tested for normality using the Lilliefors test. Since our data did not follow the normal distribution pattern, non-parametric tests were used. To assess the correlation between the endo- and exocrine dysfunction markers and the periodontal state, the Spearman's correlation coefficient was used. The differences the between the groups, as far as the hyperreactive inflammatory phenotype was concerned, were measured using the Kruskal-Wallis ANOVA, and p-values $<0.05$ were deemed statistically relevant. All calculations were performed on STATISTICA v. 10 (StatSoft, Tulsa, USA).

\section{Clinical and laboratory procedures}

Ten $\mathrm{mL}$ blood were drawn by venipuncture from every proband enrolled in the study. The serum amylase and lipase activity were measured by the automatic biochemical analyzer with colorimetric method on Cobas ${ }^{\circledR} 6000$ (Roche Diagnostics, Indianapolis, USA). A $5 \mathrm{~mL}$ EDTA whole blood sample was obtained for $\mathrm{HbA}_{1 \mathrm{c}}$ measurement, made by the turbidimetric immunoassay inhibition method on Cobas Integra ${ }^{\circledR}$ (Roche Diagnostics). All the measurements were performed in the central laboratory of the hospital adhering to the proper standardization methods set by the National Chamber of Laboratory Diagnosticians (KIDL).

The authors assumed the glycated hemoglobin blood levels $\mathrm{HbA}_{1 \mathrm{c}}$ amounting to $6.0 \%(42 \mathrm{mmol} / \mathrm{mol})$, reflecting the mean glucose blood level of $126 \mathrm{mg} / \mathrm{dL}(7.0 \mathrm{mmol} / \mathrm{L})$ as the breakpoint level of long-term control of glycemia in the blood. This coefficient was calculated based on the formula of the linear regression of blood glucose levels (1.5944 $\left.\mathrm{HbA}_{1 \mathrm{c}}[\mathrm{mmol} / \mathrm{L}]-2.5944\right)$ in accordance with the criteria of the American Diabetes Association (ADA)confirmed in 2014 by the Polish Diabetes Association (PTD) in their definition of the purpose of treatment aiming at maintaining target glycemia. ${ }^{14}$ 


\section{Results}

As a result of the clinical study, the data classifying the patients by periodontitis stage were obtained for individual study groups: patients with pancreatic adenocarcinoma, chronic pancreatitis and control group. In patients diagnosed with pancreatic cancer, the mean amylase activity was $31.9 \mathrm{IU} / \mathrm{L}$ (NS - non-significant), lipase activity 38.2 IU/L (NS) and the glycated hemoglobin 6.0\%. The highest values of the studied parameters were obtained in patients with chronic pancreatitis: the mean lipase activity was 222.9 IU/L ( $<$ 0.0001; $\mathrm{H}=45.7831)$, amylase activity $311.6 \mathrm{IU} / \mathrm{L}(\mathrm{p}=0.004 ; \mathrm{H}=55.6381)$ and the concentration of glycated hemoglobin was $8.8 \%$ ( $\mathrm{p}=0.01, \mathrm{H}=25.631$ ). Discussed results are presented in Table 1.

\section{Periodontitis activity}

The most frequently diagnosed disease entity was moderate periodontitis, which occurred in all studied groups and in the control group at the level of $44-68 \%$. The least frequent diagnosis was generalized severe periodontal disease (2-12\%), while mild periodontal disease was found in pancreatic cancer patients and in the control group (42-44\%).

In the group of patients diagnosed as suffering from chronic pancreatitis, the values of the periodontal parameters were substantially higher than in other study groups. The periodontitis activity levels measured using the BOP index amounted to $62.53 \%(\mathrm{p}=0.01 ; \mathrm{H}=61.31946)$. Moreover, the long-term periodontitis advancement ratios were the highest in this study group and amounted to a mean PD of $4.14 \pm 0.89 \mathrm{~mm}(\mathrm{p}<0.0001 ; \mathrm{H}=22.43578)$ and CAL $3.52 \pm 1.42 \mathrm{~mm}(\mathrm{p}=0.001 ; \mathrm{H}=43.97138)$. In the group of patients diagnosed with pancreatic cancer, the bleeding index BOP was estimated as $25.24 \%$ (NS), thus achieving the lowest value in the studied groups. Similarly, the mean PD ratio amounted to $2.81 \pm 0.87 \mathrm{~mm}$ (NS). In the control group the lowest value of the clinical attachment level was noted at CAL $1.61 \pm 1.02$ (NS), whereas the PD and BOP parameters amounted respectively to $3.67 \pm 1.08 \mathrm{~mm}$ (NS) and $35.32 \%$ (NS). The calculated clinical periodontitis activity level ratio was the highest in the group of patients suffering from pancreatic cancer $1.51 \pm 0.47$ ( $\mathrm{p}=0.01$; $\mathrm{H}=24.94914)$, whereas as far as patients with chronic pancreatitis were concerned, it amounted to $0.91 \pm 0.31$ (NS), and in the control group it was noted at the level of $0.65 \pm 0.16$ (NS) (Table 1).

In order to estimate the impact of oral cavity hygiene on obtained bleeding index result, the BOP/PI ratio was calculated for all studied groups. The highest value was obtained in the group of patients with pancreatic cancer -1.51 ( $\mathrm{p}=0.01 ; \mathrm{H}=24.94914$ ). The values below 1.0 were obtained in the group of patients with chronic pancreatitis and in the control group. The patients diagnosed with pancreatic cancer evinced a hyperreactive inflammatory phenotype as measured by BOP/PI inflammatory index compared with the patients diagnosed with chronic pancreatitis and the control group $(\mathrm{p}=0.01 ; \mathrm{H}=24.94914)$ (Fig. 1).

\section{Correlation between glycated hemoglobin, pancreatic lipase and amylase activity and periodontitis parameters}

In the group of patients diagnosed with chronic pancreatitis, we observed a strong positive correlation between the $\mathrm{HbA}_{1 \mathrm{c}}$ percentage and the periodontal inflammatory parameters measured clinically $(\mathrm{r}=0.31 \div 0.43$; $\mathrm{p}<0.05)$. On the other hand, there was no correlation between long-term glycemia measured by this parameter and CAL $(\mathrm{r}=0.14 ; \mathrm{p}>0.05)$. The highest correlation coefficients were achieved between the bleeding index BOP and the lipase and amylase activity in the serum amounting respectively to $\mathrm{r}=0.62$ and $\mathrm{r}=0.64$ for $\mathrm{p}<0.05$. The Spearman's correlation coefficients are presented in Table 2.

Table 1. The characteristics of the groups, taking into consideration the determination of pancreatic enzymes, HbA $\mathrm{A}_{c}$ concentration and periodontitis parameters

\begin{tabular}{|c|c|c|c|c|c|c|c|}
\hline \multirow{3}{*}{ Investigated parameters } & \multicolumn{3}{|c|}{ Condition } & \multicolumn{4}{|c|}{ Investigated groups vs control } \\
\hline & \multirow{2}{*}{$\begin{array}{c}\text { pancreatic } \\
\text { cancer }\end{array}$} & \multirow{2}{*}{$\begin{array}{c}\text { chronic } \\
\text { pancreatitis }\end{array}$} & \multirow{2}{*}{$\begin{array}{l}\text { control } \\
\text { group }\end{array}$} & \multicolumn{2}{|c|}{ pancreatic cancer } & \multicolumn{2}{|c|}{ chronic pancreatitis } \\
\hline & & & & $\mathrm{p}$-value & H-value & $p$-value & H-value \\
\hline PI [\%] & 16.96 & 69.39 & 37.81 & NS & NA & NS & NA \\
\hline $\mathrm{BOP}[\%]$ & 25.24 & $62.53^{*}$ & 35.32 & NS & NA & 0.01 & 61.31946 \\
\hline $\mathrm{PD}[\mathrm{mm}]$ & $2.81 \pm 0.87$ & $4.14 \pm 0.89^{*}$ & $3.67 \pm 1.08$ & NS & NA & $<0.0001$ & 22.43578 \\
\hline $\mathrm{CAL}[\mathrm{mm}]$ & $2.51 \pm 1.54$ & $3.52 \pm 1.42 *$ & $1.61 \pm 1.02$ & NS & NA & 0.001 & 43.97138 \\
\hline $\mathrm{HbA}_{1 \mathrm{c}}[\%]$ & 6.0 & $8.8^{*}$ & 5.6 & NS & NA & 0.01 & 25.631 \\
\hline Amylase [IU/L] & 31.9 & $311.6^{*}$ & 36.2 & NS & NA & 0.004 & 55.6381 \\
\hline Lipase [IU/L] & 38.2 & $222.9^{*}$ & 41.3 & NS & NA & $<0.0001$ & 45.7831 \\
\hline Inflammatory index & $1.51 \pm 0.47^{*}$ & $0.91 \pm 0.31$ & $0.65 \pm 0.16$ & 0.01 & 24.94914 & NS & NA \\
\hline
\end{tabular}

$\mathrm{PI}$ - plaque index; BOP - bleeding on probing; PD - probing depth; CAL - connective tissue attachment loss level; the inflammatory index was calculated as the BOP/PI ratio; ${ }^{*} \mathrm{p}<0.05$ (statistically insignificant); NS - statistically non-significant; NA - not applicable; the calculated data was presented as mean \pm standard deviation. 
Table 2. Correlation between amylase activity, lipase activity, $\mathrm{HbA}_{1 \mathrm{c}}$ percentage, and periodontal parameters in patients with chronic pancreatitis and in patients with an elevated (>6.0\%) $\mathrm{HbA}_{1 \mathrm{c}}$ level

\begin{tabular}{|l|c|c|c|c|}
\multicolumn{1}{|c|}{ Correlation } & Amylase activity & Lipase activity & $\mathrm{HbA}_{1 c} \%$ & $>6.0 \% \mathrm{HbA_{c } \%}$ \\
\hline Mean PD & $0.531730^{*}$ & $0.516398^{*}$ & $0.439446^{*}$ & $0.475526^{*}$ \\
\hline BOP & $0.643098^{*}$ & $0.625721^{*}$ & $0.379577^{*}$ & $0.632620^{*}$ \\
\hline PI & $0.483115^{*}$ & $0.514514^{*}$ & $0.310178^{*}$ & $0.602690^{*}$ \\
\hline CAL & $0.154078^{* *}$ & $0.248069^{* *}$ & $0.142726^{* *}$ & $0.502961^{*}$ \\
\hline
\end{tabular}

All numbers refer to the table refer to the Spearman's correlation coefficient; ${ }^{*} p<0.05 ;{ }^{* *} p>0.05$.
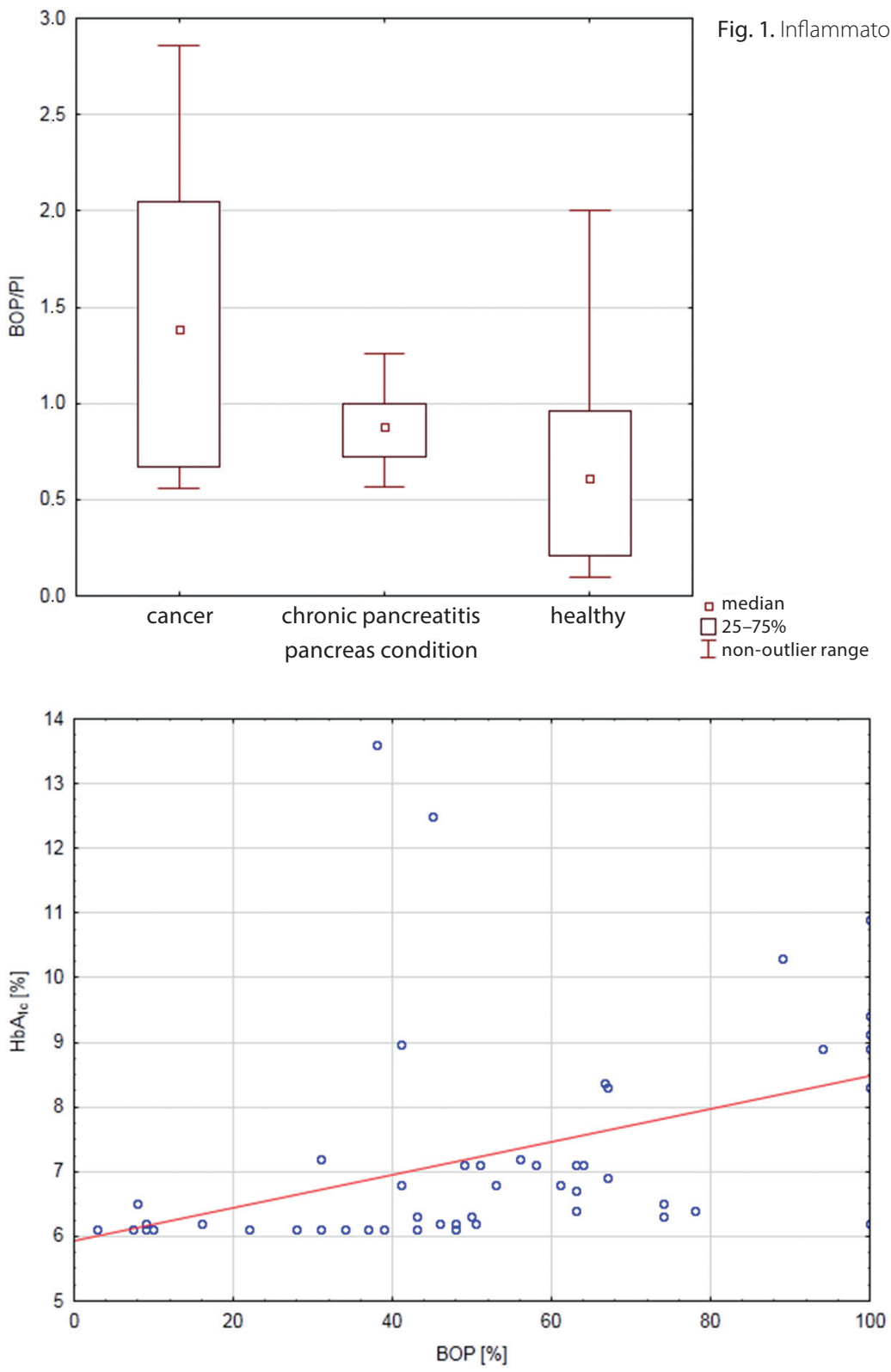

Fig. 2. Scatterplot matrix to help demonstrate the correlation between $\mathrm{HbA}_{1 \mathrm{c}}$ percentage and $\mathrm{BOP}$

(only patients with an elevated $\mathrm{HbA}_{1 c}$ level were shown)

The patients with elevated $\mathrm{HbA}_{1 \mathrm{c}}$ levels $(>6.0 \%)$ were subjected to a continued study, as far as the influence of long-term glycemia on periodontium is concerned (Fig. 2). A strong positive correlation between the immunological lesion in periodontium and hyperglycemia $(r=0.47 \div 0.63$; $\mathrm{p}<0.05$ ) was proven. Furthermore, the CAL was shown to correlate strongly with hyperglycemia in this group as well $(\mathrm{r}=0.502 ; \mathrm{p}<0.05)$ as opposed to a general sample of all patients with chronic periodontitis. The Spearman's correlation coefficients are presented in Table 2.

To separate the effects of hyperglycemia associated with an endocrine pancreatic dysfunction from the effects of its exocrine function, we searched for a correlation between the amylase activity and the periodontium state in the group of patients with well-controlled glycemia, that is with the $\mathrm{HbA}_{1 \mathrm{c}}$ percentage below $6.0 \%$ in the studied 
groups. The study findings have shown a moderately strong correlation between the lipase activity and the bleeding on probing index $(\mathrm{r}=0.347 ; \mathrm{p}<0.05)$. The study has not demonstrated any statistically relevant differences between the other periodontitis parameters and the activity of pancreatic enzymes.

\section{Discussion}

The study carried out on the population of patients diagnosed with pancreatic diseases showed a strong correlation between the clinically observed periodontitis activity levels and the pancreatic lipase and amylase activity. Groups of patients divided as per the long-term glycemia criterion were also analyzed using the glycated hemoglobin ratio. The studies conducted so far point to the influence of diabetes on the state of the periodontium. However, no data has so far been made available concerning the effects of pancreatic diseases, which may secondarily lead to uncontrolled glycemia. The authors of the study have taken both of these factors into consideration and conducted an analysis based on the diagnosis as well as on the long-term control of glycaemia $\left(\mathrm{HbA}_{1 \mathrm{c}}<6.0 \%\right)$.

Our study sheds a new light on the concomitance of periodontitis and pancreas diseases in the context of inflammatory status indices as described above. The conducted study may be indicative of mutual modulation of pancreas diseases and periodontitis. Observed positive correlation between the bleeding index BOP and the activity of pancreatic enzymes in serum may indicate a common component of inflammatory process in chronic pancreatitis and periodontal disease. The excessive bleeding observed with a small presence of local factors in patients with pancreatic cancer suggests the excessive activity of periodontitis, sometimes referred to as an overactive phenotype. So far, the cause of high values of the BOP index at a relatively low PI rate is not known, although the dysfunctions of CD14 axis receptor, $N F-\kappa B$ factors and $\mathrm{NOTCH}$ pathway proteins are postulated.

The activity of the axis mentioned above triggers a primary pathomechanism in selected systemic conditions, which consequently engages the activity of the NLRP 2 and 3 proteins mediated by $N F-\kappa B$, such as diabetes and hypertension. ${ }^{15,16}$ Therefore, the inflammation mediated by the inflammasome complex may constitute a common denominator in periodontitis and a lifestyle risk factor. In our study, we analyzed 4 basic periodontal parameters. These parameters cover various aspects of chronic inflammation encountered in periodontium. The plaque index reflects the causal factor of inflammation. The bleeding index reflects the host's vascular response in terms of hyperemia, the dilation of capillaries and increased blood flow in the region. PD and CAL refer to the long-term stages of chronic inflammation including destructive processes characteristic of a chronic inflammatory response.
The authors considered BOP in correlation with PI in the study, because their intention was to emphasize the importance of the exacerbation of a transient inflammatory response in the periodontium as a reaction to the biomass of the bacterial plaque depending on the patient's condition. An observation of this type could be a sign of an overactive phenotype of the patient noted during examination (excessive bleeding as a response to a small amount of accretions on teeth). ${ }^{17}$

The progress of chronic pancreatitis impairs both the endo- and exocrine functions of the organ. It is hard to separate these 2 processes, as they concur and intermingle. In order to do this, we selected a group of patients with well-controlled glycemia in chronic pancreatitis. In this group, as described in the results, we found a moderate correlation between the amylase activity and CAL. Although this correlation was not as prominent as the strong correlation between hyperglycemia and periodontal inflammation, this effect was clearly separable from the elevated lipase activity in the serum linked to a long-term attachment loss. All inflammatory processes, including periodontitis, are caused by a plethora of factors, and many parameters may modulate its progress. Therefore, the correlation we found in our study was far from the simple 1:1 ratio; however, the effect of exocrine deficiency alone is undeniable.

Since our study included a group of patients diagnosed with pancreatic cancer with accompanying chronic pancreatitis patients, we found it necessary to take into account the possible peculiarities concerning the inflammatory response in these patients. There was no difference in the long-term outcome of the inflammation measured by CAL. This means that the chronic inflammatory response, regardless of the underlying risk factors and modulating pancreatic factors, is similar in its final stage. However, the course and mechanisms might vary. The ratio, when elevated, shows a hyperreactive state of tissues in terms of an early inflammatory response.

\section{References}

1. Michaud DS. Epidemiology of pancreatic cancer. Minerva Chir. 2004; 59:99-111.

2. Yadav $D$, Lowenfels AB. The epidemiology of pancreatitis and pancreatic cancer. Gastroenterology. 2013;144:1252-1261.

3. Bilimoria K, Bentrem DJ, Ko CY, Stewart AK, Winchester DP, Talamonti MS. National failure to operate on early stage pancreatic cancer. Ann Surg. 2007;246:173-180.

4. Yang AL, Vadhavkar S, Singh G, Omary M. Epidemiology of alcoholrelated liver and pancreatic disease in the United States. Arch Intern Med. 2008;168:649-656.

5. Zhang L, Farrell JJ, Zhou H, et al. Salivary transcriptomic biomarkers for detection of resectable pancreatic cancer. Gastroenterology. 2010;138:949-957.

6. Wong DT, Zhang J, Farrell J, et al. Salivary biomarkers for pancreatic cancer detection. J Clin Oncol. 2009;27(Suppl 15S):4630.

7. Camen GC, Caraivan O, Olteanu M, et al. Inflammatory reaction in chronic periodontopathies in patients with diabetes mellitus: Histological and immunohistochemical study. Rom J Morphol Embryol. 2012;53:55-60. 
8. Yoon AJ, Cheng B, Philipone E, Turner R, Lamster IB. Inflammatory biomarkers in saliva: Assessing the strength of association of diabetes mellitus and periodontal status with the oral inflammatory burden. J Clin Periodontol. 2012;39:434-440.

9. Wong JC, Raman S. Surgical resectability of pancreatic adenocarcinoma: CTA. Abdom Imaging. 2010;35:471-480.

10. Etemad B, Whitcomb DC. Chronic pancreatitis: Diagnosis, classification, and new genetic developments. Gastroenterology. 2001;120: 682-707.

11. Tanaka S, Nakaizumia A, loka T, et al. Main pancreatic duct dilatation: A sign of high risk for pancreatic cancer. Jpn J Clin Oncol. 2002;68: 237-242.

12. Petrone MC, Arcidiacono PG, Testoni PA. Endoscopic ultrasonography for evaluating patients with recurrent pancreatitis. World J Gastroenterol. 2008;14:1016-1022.
13. Mombelli A. Clinical parameters: Biological validity and clinical utility. Periodontol 2000. 2005;39:30-39.

14. Nathan DM, Kuenen J, Borg R, et al. Translating the A1C assay into estimated average glucose values. Diabetes Care. 2008;31:14731478.

15. Wali JA, Masters SL, Thomas HE. Linking metabolic abnormalities to apoptotic pathways in Beta cells in type 2 diabetes. Cells. 2013;2: 266-283.

16. Yin Y, Pastrana JL, Li X, et al. Inflammasomes: Sensors of metabolic stresses for vascular inflammation. Front Biosci. 2013;18:638-649.

17. Kornman KS, Crane A, Wang HY, et al. The interleukin-1 genotype as a severity factor in adult periodontal disease. J Clin Periodontol. 1997;24:72-77. 